\title{
Fever of unknown origin: A prospective study in Northern Iraq
}

\author{
Rami M. Adil Khalil*, Rafe' H. Al-Kazzaz ${ }^{* *}$, Humam Ghanim**, Dhia J. Al-Layla* \\ * Department of Medicine, College of Medicine, University of Mosul; ** Department of Medicine, Ibn Sina \\ Teaching Hospital; *** Department of Community Medicine, College of Medicine, University of Mosul.
}

(Ann. Coll. Med. Mosul 2010; 36 (1 \& 2): 26-35).

Received: $9^{\text {th }}$ Jun 2010; Accepted: $9^{\text {th }}$ Feb 2011.

\begin{abstract}
Objectives: A wide variety of diseases are likely causes of fever of unknown origin (FUO). No fixed guidelines exist to direct the workup in these cases. We followed a diagnostic protocol to study the causes of FUO in Iraq, and to evaluate the contribution of clinical assessment and various investigations in making the final diagnosis.

Methods: From March 2002 to September 2009, fifty five consecutive patients with FUO were admitted in a tertiary referral centre in Mosul, Iraq. The patients underwent a series of clinical and diagnostic evaluation in a prospective study, in an attempt to diagnose the underlying cause of fever. The benefit of history taking and clinical examination as directors of the diagnostic workup and the yield of various laboratory and imaging techniques were assessed.

Results: Infections were the commonest causes of FUO (32.7\%), followed by non-infectious inflammatory diseases (NIID) (25.4\%), malignancies (16.4\%) and miscellaneous causes $(5.4 \%)$. No diagnosis was made in $20 \%$ of cases. Of infections, tuberculosis was the most important single cause of fever, while various vasculitides and non-Hodgkin's lymphoma were the commonest NIID and malignant disease, respectively. Symptoms of the patients were of little benefit in directing subsequent investigations, but the physical signs were more useful; finding enlarged lymph nodes was significantly associated with malignant diseases $(p=0.009)$. Anaemia, high ESR and elevated liver enzymes were common and bear no significant association with any disease category. Chest radiograph and abdominal ultrasound were helpful initial imaging studies. CT scan of the chest was shown a useful diagnostic procedure.

Conclusion: Together with infections, NIID are important causes of FUO in Iraq. Careful physical examination and a systematic approach on investigations are usually rewarding in reaching the diagnosis.
\end{abstract}

\section{الخلاصة}

الأهداف: أمر اض مختلفة قد تكون سببا للحمى مجهولة المصدر. لا نوجد خطوط هادية محددة لتوجيه العمل التشخيصي في مثل هذه الحالات. اتبعنا منهجا تشخيصيا لدراسة حالات الحمى مجهولة المصدر في العراق، ولتقييم ما تسهم به

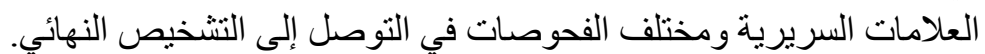

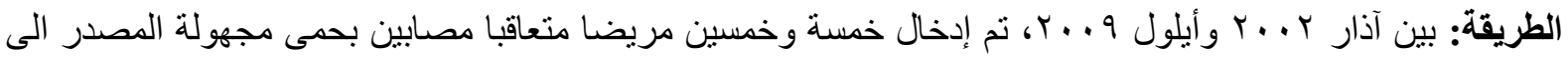
مركز إحالة من المرتبة الثالثة في الموصل شمالي العراق. خضع المرضى لسلسلة من الفحوصات السريرية و المختبرية

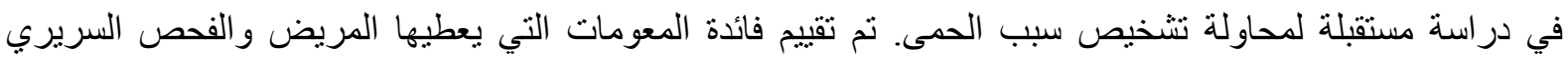
كموجهات للعمل التشخيصي وحصيلة مختلف الفحوصات المختبرية والتصويرية التي تجرى في محاولة الكثف عن التشخيص النهائي.

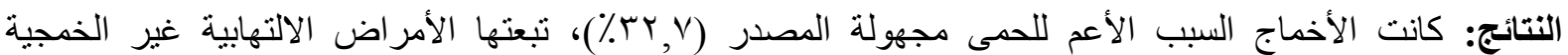

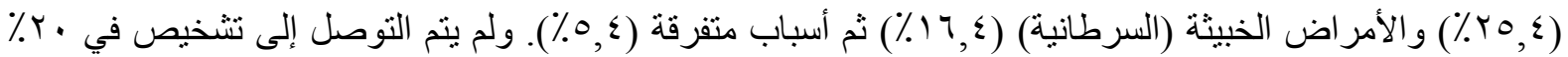




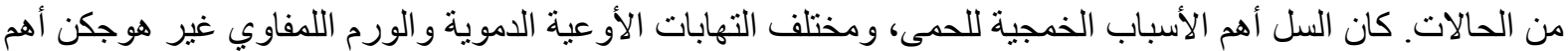

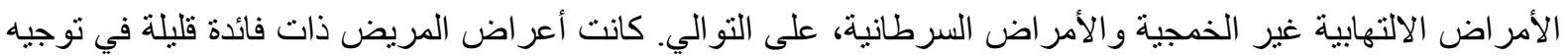
الفحوصات المختبرية، إلا أن الفحص السريري كان أكثر فائدة، حيث أن تضخم العقد اللمفاوية كان علامة دالة على التى الكان

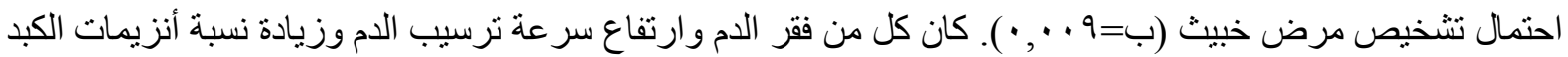

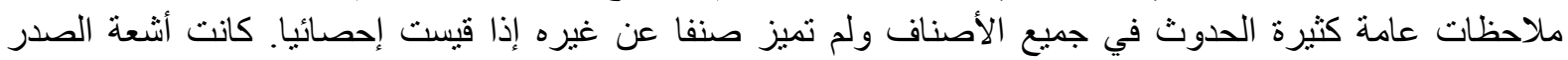
وفحص البطن بالأمواج فوق الصوتية دراستين تشخيصيتين مفيدتين. ظهر مفر اس الصدر كإجر اء تثخيصي فعال في مثل هذه الحالات. الاستتتاج: إضافة إلى الأخماج، كانت الأمر اض الالتهابية غير الخمجية سببا مهما للحمى مجهولة المصدر في العراق.

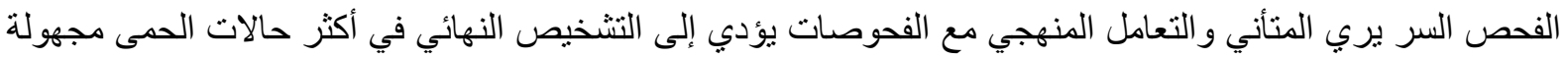

$\mathrm{F}$ ever of unknown origin (FUO) is one of the most challenging problems that can face a physician during his clinical practice. The earliest work on this subject was published by Petersdorf and Beeson in $1961^{(1)}$. They defined FUO as fever of at least 3 weeks duration exceeding $38.3^{\circ} \mathrm{C}$ on several occasions, with no established diagnosis after one week of evaluation in hospital. Considering the increased cost of in-hospital care and advances in investigative techniques that accelerated the pace of exploration, Durak and Street ${ }^{(2)}$ (agreed by Petersdorf ${ }^{(3)}$ ) revised the conventional definition in 1991, limiting the hospital stay to 3 days or more than two outpatient's visits. They also divided FUO into four groups: classic, neutropenic, nosocomial and HIV related FUO.

The spectrum of diseases responsible for FUO varies in different geographical locations and changes over time in the same locality ${ }^{(4,5)}$. The aim of our work was to study the causes of classic FUO in Iraq and to evaluate the contribution of clinical assessment and various investigations in making the diagnosis.

\section{Patients and methods:}

From March 2002 to September 2009, we gathered a series of consecutive patients with prolonged fever presented to Ibn Sina Teaching Hospital in Mosul Medical City (Mosul, Iraq), and admitted under the care of one of the first two authors. Patients were referred from general practitioners and family physicians. Some of them presented directly without referral, or came to our attention after consultations from other physicians. Patients were included in the study if they fulfilled the Durak and Street's revised criteria of classic FUO. Accordingly, patients should be at least 10 years old, and have documented fever of $38.3^{\circ} \mathrm{C}$ or more, which was repeated on more than one occasion, for more than 3 weeks, with no diagnosis after 3 days of hospital admission or two outpatients' visits.

Patients were excluded from the study if the diagnosis was already established before referral. Patients whose fever has developed in hospital (nosocomial), had severe neutropenia (defined as WBC count $<1.0 \times$ $10^{9} / \mathrm{L}$, or granulocyte count $<0.5 \times 10^{9} / \mathrm{L}$ ) or HIV infection were also excluded.

Fifty five (55) patients met the above criteria, and after taking their informed consent, they were included in a prospective study considering the final diagnosis as the main outcome measure.

The final diagnosis was either made during hospital admission or during the follow up period. Only diagnoses that were sufficiently validated were retained. The test most convincingly ascertained the final diagnosis in each case was determined. Patients were labelled as "no diagnosis" if they died during the evaluation period or remained undiagnosed during their hospital stay and subsequent follow up period, which was open until the end of the study.

The causes of FUO were classified into 4 categories: 1. infection, 2. malignancies, 3. non-infectious inflammatory diseases (NIID) (a category coined by de Kleijn et al in 1997 that 
comprises connective tissue diseases, vasculitis and granulomatous diseases ${ }^{(6)}$ ) and 4. miscellaneous causes which do not fit in any of the above categories.

A comprehensive review of history was done, stressing on the presence of localizing symptoms, with careful consideration of past medical and surgical history, details of travel, occupation and animal exposure. Careful clinical examination was conducted by the authors themselves. Some patients were referred for clinical evaluation by specialists in other fields; namely, surgeons, otolaryngologists, ophthalmologists, dermatologists, neurologists and gynaecologists. Patients were re-examined daily during their admission and on each follow up visit for possible appearance of new signs that may help in reaching the diagnosis. Any available records of examination, medications or investigations were reviewed. All patients underwent an initial diagnostic evaluation (step 1 investigations). These are mentioned in table 1.

If the diagnosis had not been established after the initial clinical and laboratory evaluation, investigations from the second diagnostic set were started (step 2 investigations (table 2)). No rigid protocol was followed, as no published guidelines exist on the approach of $\mathrm{FUO}^{(7)}$. The strategy suggested by Knockaert in 1992 was considered $^{(8)}$. Selection of step 2 investigations was based on the presence of potentially diagnostic clues from history, examination and step 1 investigation. Much weight was given to exclude the common diseases in the community.

If the diagnosis was still illusive after this step, a third stage investigation was considered (step 3 investigations (table 2)). These included invasive procedures (like liver biopsy, laparoscopy or surgical exploration) or therapeutic trials. The latter were only conducted when the patient was otherwise deteriorating; the choice was dictated by clinical suspicion, this mostly involved antituberculosis therapy for suspected cryptic tuberculosis and corticosteroid therapy when certain NIID was highly likely in the absence of diagnostic laboratory test (such as the case with Still disease).

Table (1): Step 1 obligatory investigations.

\section{Step 1 investigations}

1. Complete blood picture and ESR.

2. General urine examination.

3. Serum urea, creatinine and electrolytes.

4. Liver function tests (bilirubin, alanine and aspartate transaminases, alkaline phosphatase and albumin).

5. Blood and urine culture.

6. Brucella agglutination test, Widal test and hepatitis serology.

7. Antinuclear antibodies, rheumatoid factor.

8. Chest X-ray.

9. Ultrasound of the abdomen and pelvis.

Table (2): Step 2 and 3 investigations.

\section{Step 2 investigations}

1. Other biochemical tests: prostate specific antigen (PSA), acid phosphatase, T3, T4, $\mathrm{TSH}$, protein electrophoresis, creatine kinase, troponins.

2. Serological tests: antiDS antibodies, antineutrophil cytoplasmic antibodies (ANCA).

3. Blood film for malaria.

4. Bone marrow aspirate (or biopsy) and culture.

5. CT scan of the chest, abdomen and pelvis.

6. lumbar puncture and CSF examination.

7. MRI of the brain, spine or abdomen.

8. Echocardiography.

9. Endoscopy (gastroscopy, colonoscopy or bronchoscopy).

10. Biopsy from pathological specimens (pleural, endoscopic, lymph nodes... etc.).

\section{Step 3 investigations}

1. Liver biopsy.

2. Laparoscopy.

3. Surgical exploration.

4. therapeutic trial. 
The data were tabulated and analyzed using MiniTab version (13.20). Z two - proportions with $95 \%$ confidence interval was used in comparing different proportions. $\mathrm{P}$ - value < 0.05 was considered significant throughout the study.

\section{Results}

Of the 55 patients included in the study, 27 were males and 28 were females $(49.1 \%$ and $50.9 \%$ respectively). The patients mean age was $42.76 \pm 19.29$ years, ranging from $10-76$ years. Excluding 5 patients with long history ( $>6$ months) of intermittent fever, the mean duration of fever was $43.28 \pm 34.35$ days.

Infection was the most common cause of FUO, responsible for 18 cases (32.7\%). NIIDs were diagnosed in 14 patients (25.4\%) and malignancies in 9 patients $(16.4 \%)$. Miscellaneous causes were found in 3 patients $(5.4 \%)$. No diagnosis was made in 11 patients (20\%); four (4) of them died during evaluation (table 3).

Tuberculosis was the infection most frequently responsible for FUO in our study. Only one of the 4 patients had pulmonary tuberculosis, in whom a faint apical cavitary lesion was overlooked in the earlier films. The others had cryptic tuberculosis (diagnosed after therapeutic trial), disseminated disease (affecting lumbar spines, meninges and ovaries) and pericardial disease. Typhoid and brucellosis were diagnosed in 3 patients each. All the typhoid patients had received multiple courses of antibiotics before presentation and their blood cultures were negative. All of them had splenomegaly and two had neutropenia. The diagnosis was confirmed by bone marrow culture in one patient and by rising titre of Widal test, exclusion of other diseases and a response to treatment in the other two. splenomegaly was noticed in two out of the three patients with brucellosis. The diagnosis was established by a positive brucella agglutination test (which was negative on preadmission testing). Two patients were diagnosed as infective endocarditis confirmed by a positive blood culture (growing Staphylococcus aureus in both cases) and echocardiographic evidence of vegetations. In two patients, urinary tract infection was determined to be the cause of fever. Both had pyuria on urine examination, with ultrasonographic features of acute pyelonephritis. Urine culture was positive for $E$. coli in one of them only (the diagnosis was supported by a positive response to treatment in the other). A woman with ovarian abscess was diagnosed after surgical exploration for suspected ovarian tumour. Malaria was confirmed by a positive blood film after two negative results. An elderly woman with inconclusive chest $\mathrm{X}$-ray was diagnosed with basal pneumonia on CT scan of the chest, and a young man had infectious mononucleosis.

Table (3): Causes of fever of unknown origin in 55 patients.

\begin{tabular}{|l|l|}
\hline 1. Infections & $18(32.7 \%)$ \\
Tuberculosis & $4(7.3 \%)$ \\
Typhoid fever & $3(5.5 \%)$ \\
Brucellosis & $3(5.5 \%)$ \\
Infective endocarditis & $2(3.6 \%)$ \\
Pyelonephritis & $2(3.6 \%)$ \\
Pneumonia & $1(1.8 \%)$ \\
Malaria & $1(1.8 \%)$ \\
Ovarian abscess & $1(1.8 \%)$ \\
Infectious mononucleosis & $1(1.8 \%)$ \\
2. Non-infectious inflammatory diseases & $14(25.4 \%)$ \\
Systemic lupus erythematosus & $2(3.6 \%)$ \\
Wegener's granulomatosis & $2(3.6 \%)$ \\
Polyarteritis nodosa & $1(1.8 \%)$ \\
Microscopic polyangiitis & $1(1.8 \%)$ \\
Temporal arteritis & $1(1.8 \%)$ \\
Behçet's syndrome & $1(1.8 \%)$ \\
Mixed connective tissue disease & $1(1.8 \%)$ \\
Adult onset Still disease & $1(1.8 \%)$ \\
Ulcerative colitis & $1(1.8 \%)$ \\
Crohn's disease & $1(1.8 \%)$ \\
Familial Mediterranean fever & $1(1.8 \%)$ \\
Subacute thyroiditis & $1(1.8 \%)$ \\
3. Malignant diseases & $9(16.4 \%)$ \\
Non-Hodgkin's lymphoma & $3(5.5 \%)$ \\
Carcinoma of the prostate & $2(3.6 \%)$ \\
Hodgkin's disease & $1(1.8 \%)$ \\
Carcinoma of the colon & $1(1.8 \%)$ \\
Multiple myeloma & $1(1.8 \%)$ \\
Liver metastases (unknown primary) & $1(1.8 \%)$ \\
4. Miscellaneous causes & $3(5.4 \%)$ \\
Liver cirrhosis (hepatitis B) & $2(3.6 \%)$ \\
Migraine & $1(1.8 \%)$ \\
5. No diagnosis & $11(20 \%)$ \\
Persistent fever & $7(12.7 \%)$ \\
Death & $4(7.3 \%)$ \\
\hline
\end{tabular}


Systemic lupus erythematosus was responsible for the FUO in two patients and mixed connective tissue disease in a third one. Adult onset Still disease was diagnosed in a patient with one year of prolonged intermittent fever, generalized lymphadenopathy (including intra-abdominal lymph nodes), splenomegaly and skin rash. After negative lymph node biopsy, the patient dramatically responded to corticosteroid therapy albeit with recurrent relapses over three years follow up. Vasculitides (including Behçet's syndrome) were responsible for six cases. Two patients were proved to have ANCA positive Wegener's granulomatosis (one presented with biopsy confirmed granulomatous mastitis and pulmonary cavitary lesions missed as tuberculosis, and one with cavernous sinus thrombosis, pulmonary infiltrate and pleural effusion). A woman was treated empirically with corticosteroids after extensive cavitating nodular lesion was diagnosed as vasculitis on CT scan. Complete resolution and long time remission on immunosuppressive therapy was noticed. Polyarteritis nodosa was diagnosed on clinical basis in a young man with hypertension, mononeuritis multiplex, skin nodules, vaculitic skin rash and ischaemic stroke. Prolonged remission (4 years) was attained on steroids and immunosuppressants. After unexplained fever, Behçet's syndrome was diagnosed when a patient developed oligoarthritis and posterior uvietis on follow up visits, Pethargy test was also positive. The sixth case of vasculitis was an elderly woman with temporal arteritis associated with polymyalgia rheumatica. Two cases of inflammatory bowel disease were diagnosed; one with mild diarrhoea, in whom ulcerative colitis was diagnosed by colonoscopy and biopsy; and the second with Crohn's disease suspected on clinical and radiological grounds and proved after exploration for colonic perforation. In a patient with prolonged episodic fever and hepatosplenomegaly, liver biopsy diagnosed amyloidosis, with retrograde diagnosis of familial Mediterranean fever. A woman with fever, high ESR and mild thyrotoxicosis was diagnosed as subacute thyroiditis by needle aspiration of the thyroid, with dramatic response to corticosteroids.

Lymphoma was the main malignancy responsible for FUO in our cohort. All four cases were confirmed by lymph node biopsy. Non-Hodgkin's lymphoma was found in three patients, two with mixed small and large cell type B cell lymphoma, and one with angioimmunoblastic $\mathrm{T}$ cell lymphoma (formally angioimmunoblastic lymphadenopathy). The fourth was a patient with Hodgkin's disease who was diagnosed quite late, as initial evaluation wrongly suggested infective endocarditis, but the patient failed to respond to antibiotic therapy. One month later cervical lymphadenopathy appeared and permitted the correct diagnosis to be made. Unusually two of our patients were shown to have carcinoma of the prostate with bone metastases (one of them discovered by CT scan). Acid phosphatase was markedly elevated in both, but prostate specific antigen was abnormal in one of them only. Carcinoma of the colon was diagnosed by colonoscopy after prolonged fruitless investigation of a patient with fever, abdominal pain and constipation, but otherwise negative imaging studies. Fever was caused by multiple myeloma and by metastases to the liver and spleen of unknown primary in two other patients.

Another patient with prolonged history of paroxysmal fever in association with headache and neck pain was clinically diagnosed as migraine. The diagnosis was confirmed by durable response to propranolol prophylaxis and recurrent relapses on its withdrawal. Unexpectedly, two patients were found to have liver cirrhosis due to hepatitis $B$ infection. The first had negative laparoscopic liver biopsy despite nodular liver on MRI and CT scan. Gastroduodenoscopy has been initially normal, but the patient bled heavily from oesophageal varices few months later. The other one presented with hepatosplenomegaly and abnormal liver function tests in association with fever. The diagnosis was established when further evaluation disclosed ascites and oesophageal varices.

Beyond fever and associated constitutional symptoms of fatigue, malaise, sweating and 
generalized aches, 19 patients (34.6\%) described symptoms that potentially localize the source of fever. In only 6 of these patients $(31.6 \%)$, their symptoms really led to the true diagnosis.

No finding apart from fever was noticed on clinical examination of 10 patients $(18.2 \%)$, while positive findings were demonstrable in the remaining 45 patients $(81.8 \%)$. In 11 patients, new clinical signs appeared during hospital stay or follow up visits. In 6 of them, discovery of these signs contributed to the final diagnosis. Diagnosis was made in 38 out of 45 patients with positive clinical examination $(84.4 \%)$, and in 6 out of 10 patients with negative clinical examination $(60 \%) \quad(95$ percent confidence interval, $(0.177$ to 1.06 ; $P=0.136$ ). Finding lymph node enlargement on physical examination was significantly associated with subsequent diagnosis of malignant disease $(p=0.009)$. No similar relationship was noticed with splenomegaly $(p=0.131)$.

None of the obligatory biochemical investigations revealed the diagnosis by its own; however, many of these tests were helpful as potentially diagnostic clues. Elevated serum urea and creatinine pointed to renal impairment that was relevant four out of five times. Uraemia was an initial marker of amyloidosis, vasculitis, carcinoma of the prostate and multiple myeloma. Transaminases and alkaline phosphatase were elevated in $12(21.8 \%)$ and $17(31 \%)$ patients, respectively, but they did not show a particular association with any diagnostic category.

The majority of patients were anaemic (69.1\%). All (except two with hypochromic anaemia) were having normochromic normocytic anaemia. Malignancy was more commonly diagnosed among anaemic patients than among those without anaemia (21.1\% versus 5.9\%) (95 percent confidence interval, 0.019 to $0.322 ; p=0.082$ ). Mean ESR was not significantly different between the diagnostic groups. Although an ESR of $100 \mathrm{~mm} / \mathrm{h}$ or higher was more commonly noticed in patients with malignancy and NIID (44.4\% and $41.7 \%$ respectively) than in patients with infections
$(22.2 \%)$, this difference was not statistically significant (95 percent confidence interval, (0.400 to $0.455 ; p=0.899$ ).

Blood culture was diagnostic in two cases of staphylococcus aureus infective endocarditis, but positive cultures were misleading in 6 patients (growing staphylococci and $\alpha$ haemolytic streptococci) in patients ultimately found to have unrelated diagnosis. On the other hand, bone marrow culture diagnosed one patient with typhoid fever and supported positive blood culture result in a patient with infective endocarditis.

Chest X-ray and ultrasound examination of the abdomen and pelvis were ordered routinely as step 1 investigation. Chest X-ray was abnormal in $15(27.3 \%)$ patients, and provided useful findings that led to the correct diagnosis in 7 of them (diagnostic yield of $12.7 \%$ ), while ultrasound showed abnormal findings in 36 patients $(65.6 \%)$, and truly contributed to the final diagnosis in 16 patients (diagnostic yield of $29 \%$ ). CT scan of the abdomen (ordered in 16 patients) added useful information beyond ultrasound findings in 2 cases only, while CT of the chest was diagnostically useful beyond chest X-ray in 4 out of 5 patients $(80 \%)$.

\section{Discussion}

Despite the enormous improvement in the diagnostic abilities provided by the new techniques of investigations, the percentage of undiagnosed cases of FUO has steadily increased over the last decades, rising from a mean of $17.2 \%$ in 1970 s to $30 \%$ in $1990 \mathrm{~s}^{(9)}$. One explanation is that patients nowadays tend to seek medical advice early and that a diagnosis is frequently established before 3 weeks elapsed ${ }^{(10)}$. Only the difficult, hard to diagnose cases would remain. Moreover, advanced diagnostic techniques helped to diagnose many cases which were previously considered as FUO, with selection of difficult to diagnose cases, mostly self limiting or benign fevers. The percentage of undiagnosed cases in our study is intermediate among those of recent studies (table 4).

We followed a diagnostic protocol that made use of the previous experience in this field, with two considerations regarding infectious 
disease investigations: 1 . We avoided blind microbiological serological screening because such strategy was associated with a very low diagnostic yield ${ }^{(11)}$. 2. Studies in many countries have shown that the spectrum of infections presenting as FUO in a community is a mirror reflection of the endemic infections in that community. Among infections, $Q$ fever was an important cause of FUO in France ${ }^{(12)}$, leishmaniasis in India ${ }^{(13)}$, melioidosis in Taiwan $^{(14)}$ and brucellosis in Jordan ${ }^{(15)}$ and Turkey ${ }^{(16)}$. Accordingly, we adapted the study algorithm to the epidemiological status in Iraq and included brucella agglutination test and Widal test in the initial diagnostic workup.

Until recently, it was almost a constant finding in all studies of FUO that infections represent the major category of cases. In series from more developed countries (USA and Europe $3,6,10,-12$ ), the proportion of cases diagnosed as infections has gradually declined over the last four decades, with a parallel rise in NIID. In some countries, like France ${ }^{(12)}$ and Belgium ${ }^{(10)}$, these diseases are now the leading cause of FUO. Infection is still the dominating category in FUO series from developing countries $^{(13,14.16-19)}$. Comparing the distribution of FUO cases in the different categories in studies published over the last 5 years (table 4 ), the proportion of infections in our present study was less than that of other developing countries and nearer to the figures of European studies.

As much as high percentage of infectious diseases reflects the high prevalence of infection in that community, it is also true that a high rate of infectious diseases presenting as FUO indicates a diagnostic problem at the outpatient level as far as most cases represent atypical presentation of common diseases ${ }^{(1,10)}$. The early consideration of diseases like typhoid fever and brucellosis by general practitioners has probably contributed to the relatively small number of cases due to infection in our study. Tuberculosis was the leader among infections that were responsible for FUO in developing countries (ranging from $35.5 \%-45.2 \%$ of all infections ${ }^{(13,15,22,23)}$ ). Although it was the commonest infection in our series, the proportion of these cases $(22.2 \%)$ was less than other studies showed. Taking in consideration that tuberculosis is highly endemic in Iraq, this was an unusual finding. A possible explanation is the trend toward early introduction of anti-tuberculosis therapy for any difficult to explain febrile illness before referring them as FUO.

The group of diseases not caused by infection or neoplasm had different nomenclature in FUO series: inflammatory, connective tissue diseases, collagen vascular disease. . etc. Since the adoption of the name "non infectious inflammatory diseases (NIID)" by de Kleijn et $\mathrm{al}^{(6)}$, subsequent studies have used this term in their work. Similar to other studies, NIID has emerged as an important cause of FUO in Iraq, with a particularly high percentage of cases caused by a variety of vasculitides which comprised $10.9 \%$ of overall cases.

In the diagnostic category of malignancy, non-Hodgkin's lymphoma was the main cause of FUO, a finding that is shared by most other studies in the field ${ }^{(6,10,13,15-17,22,24)}$. Although carcinoma of the prostate has been reported to cause $\mathrm{FUO}^{(25)}$, such cases are rare, and it was strange to diagnose two cases in one series.

Table (4): The percentage of patients with FUO by cause in the most recent studies.

\begin{tabular}{|c|c|c|c|c|c|c|c|c|}
\hline & $\begin{array}{c}\text { Italy } \\
\text { (Mansueto) }^{20}\end{array}$ & $\begin{array}{c}\text { China } \\
(\text { Zheng })^{21}\end{array}$ & 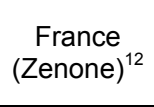 & $\begin{array}{c}\text { Iraq } \\
\text { (Present } \\
\text { study) }\end{array}$ & $\begin{array}{l}\text { Taiwan } \\
(\text { Chin })^{14}\end{array}$ & $\begin{array}{c}\text { India } \\
(\text { Kejariwal })^{13}\end{array}$ & $\begin{array}{c}\text { Mexico } \\
\text { (Avec- } \\
\text { Salinas) }^{17}\end{array}$ & $\begin{array}{c}\text { Turkey } \\
\text { (Coplan) }^{16}\end{array}$ \\
\hline Infections & 31.8 & 49.1 & 22.9 & 32.7 & 57.4 & 53 & 42.2 & 45.1 \\
\hline NIID & 12.2 & 7.8 & 26.4 & 25.4 & 7.8 & 11 & 26.7 & 26.8 \\
\hline Malignancy & 14.2 & 7.8 & 9.7 & 16.4 & 8.5 & 17 & 17.8 & 14.1 \\
\hline Miscellaneous & 9.2 & 8.8 & 15.3 & 5.4 & 8.5 & 5 & 1.2 & 5.6 \\
\hline Undiagnosed & 31.8 & 26.5 & 25.7 & 20 & 17 & 14 & 11 & 8.5 \\
\hline
\end{tabular}


Although many studies stressed on the importance of careful history and clinical examination in directing FUO workup ${ }^{(5-7)}$, symptoms described by the patients were potentially localizing in only one third of cases. These proved falsely localizing in $69.4 \%$ of cases, and did not lead to the correct diagnosis. This misleading symptomatology was probably one factor that contributed to delayed diagnosis in these patients. Careful physical examination on the other hand can be rewarding. Diagnosis was more likely to be made when there were positive clinical signs that would direct the subsequent investigations (successful diagnosis in $84.4 \%$ of patients with positive clinical examination versus $60 \%$ in those without). De Kleijn was able to diagnose $70 \%$ of patients ${ }^{(6)}$ with potentially diagnostic clues and $60 \%$ of those lacking such clues. But the difference was not statistically significant, neither in our study, nor in De Kleijn's. The presence of enlarged lymph nodes was found a predictor of subsequent diagnosis of malignant disease, supporting similar notice made by Coplan et $\mathrm{al}^{(16)}$. However, the association between splenomegaly and malignancy as a cause of FUO has not been confirmed in this study.

Liver function tests (manifested as serum transaminases and/or alkaline phosphatase) were abnormal in $38.2 \%$ of cases. Such changes were not related to a particular disease category and they represented a real liver disease in only one patient $(1.8 \%)$. Other studies also noticed non-specific elevation of liver enzymes in $50 \%$ of cases ${ }^{(11)}$. In a second study ${ }^{(16)}$, high serum aspartate transaminase (AST) was found to be associated with NIID. Such relationship was not confirmed in our study.

Regardless to the diagnostic category, most of our patients were anaemic $(69.1 \%)$; with normochromic normocytic blood picture. Similar percentage of anaemia was found by de Kleijn et al $(70 \%)^{(6)}$, while $97.5 \%$ of Gupta et al patients were anaemic ${ }^{(26)}$.

In agreement with Coplan et $\mathrm{al}^{(16)}$, mean ESR was not statistically different between the groups. An ESR equal to or exceeding 100 was not found a statistically significant pointer of a particular aetiology.

The diagnostic yield of chest X-ray and ultrasound of the abdomen and pelvis were high enough to include them in the initial step of evaluation. Although CT scan of the abdomen has been reported to have a diagnostic yield of $19 \%$ in the workup of $\mathrm{FUO}^{(9)}$, ultrasound of the abdomen possessed a similar ability to detect local pathology. In a recent study, standardized ultrasound examination correctly diagnosed the cause of fever in one third of FUO cases ${ }^{(27)}$, a result that is comparable to our findings. Considering the costs of the two studies and the risk of radiation associated with $\mathrm{CT}$ scan, ultrasound was a logical starting investigation, which can be supplemented by CT scan in case of negative or indeterminate results.

CT scan of the chest has not been studied well in the investigation of FUO cases. Out of five times this test was ordered, useful diagnostic findings were obtained in four of them. These findings support the recommendation of Lopez et al to include CT scan of the chest as well as the abdomen in the investigation strategy of $\mathrm{FUO}^{(28)}$.

It is important to note that polymerase chain reaction $(P C R)$ is gaining increasing utility in the diagnosis of many infectious diseases that may present as FUO. Of particular interest was the ability of PCR to diagnose malaria in patients with low parasitaemia and thus negative blood film ${ }^{(29)}$. PCR in samples of bone marrow aspirate diagnosed tuberculosis in $33 \%$ of patients clinically suspected to have the disease, despite negative bone marrow culture. Subsequent improvement on antituberculosis drugs was shown in $85 \%$ of these patients $^{(30)}$. Nested PCR (nPCR) targeting the flagellin gene (fliC) of salmonella typhi was carried out on DNA extracted from the buffy coat layer of blood samples. The test had sensitivity and specificity of $100 \%$ and $97.5 \%$, respectively. In a study from rural India, nPCR diagnosed typhoid fever in $4.2 \%$ of cases of FUO, while concomitant blood culture (using completely automated blood culture system) diagnosed the disease in $2 \%$ of cases only ${ }^{(31)}$. 


\section{Conclusion}

Although infections are still the commonest cause of FUO in Iraq, NIIDs are important causes and should always be considered. Systematic approach for the diagnosis of cases of FUO is usually rewarding.

\section{References}

1. Petersdorf RG, Beeson PB. Fever of unexplained origin: report on 100 cases. Medicine (Baltimore).1961;40:1-30.

2. Durack DT, Street AC. Fever of unknown origin - re-examined and redefined. Curr Clin Top Infect Dis. 1991;11:35-51.

3. Petersdorf RG. Fever of unknown origin: an old friend revisited. Arch Intern Med. 1992; 125:21-224.

4. Barbado FG, Vasquez JJ, Pina JM, Arnalich F, Ortiz - Vasquez J. Pyrexia of unknown origin: changing spectrum of diseases in two consecutive series. Postgrad Med J. 1992;68:884-887.

5. Murray MF, Tomford JW, Rehm SJ, McHenry MC, Keys TF, Longworth DL. The changing spectrum of fever of unknown origin (FUO): A review of 106 cases at a referral hospital [abstract]. Abstracts of the 1993 IDSA Annual Meeting. Abstract 238, p 570, 1993.

6. de Kleijn EM, Vandenbrouch JP, van der Meer JW. I. Fever of unknown origin (FUO): A prospective multicenter study of 167 patients with FUO, using fixed epidemiologic entry criteria. Medicine (Baltimore).1997;76:392-400.

7. Tolia J, Smith LG. Fever of unknown origin: historical and physical clues to making the diagnosis. Infect Dis Clin North Am. 2007;21(4):917-36, viii.

8. Knockaert DC. Diagnostic strategy for fever of unknown origin in the ultrasonography and computed tomography era. Acta Clin Belg. 1992; 47(2): 100-16.

9. Mourad O, PaldaV, Detsky A. A comprehensive evidence based approach to fever of unknown origin. Arch Intern Med. 2003; 163:545-551.

10. Vanderschueren $S$, Knockaert D, Andriaenssens T, Demey W, Durnez A,
Blockmans D, Bobbaers H. From prolonged febrile illness to fever of unknown origin: The challenge continues. Ann Intern Med. 2003;163:1033-1041.

11. de Kleijn EM, Vandenbrouch JP, van der Meer JW. II. Diagnostic procedures in a prospective muticenter study of 167 patients. Medicine (Baltimore). 1997; 76: 401-414.

12. Zenone T. Fever of unknown origin in adults: Evaluation of 144 cases in a nonuniversity hospital. Scand J Infect Dis. 2006; 38(8):632-638.

13. Kajariwal D, Sarkar N, Chakraboti SK, Agarwal V, Roy S. Pyrexia of unknown origin: A prospective study of 100 cases. J Postgrad Med. 2001;47:104-107.

14. Chin C, Chen YS, Lee SS, Wann SR, Lin $\mathrm{HH}$, Lin WR, Huang CK, Tsai $\mathrm{HC}$, Kao CH, Yen MY, Liu YC. Fever of unknown origin in Taiwan. Infection. 2006;34(2):75-80.

15. Ammari F. Fever of unknown origin in North Jordan. Trop Doct. 2006;36:251-3.

16. Coplan A, Onguru P, Erbay A, Akinci E, Cevik M, Erin $S$, Bodur $H$. Fever of unknown origin: Analysis of 71 consecutive cases. Am J Med Sci. 2007; 334: 92-96.

17. Arce-Salinas CA, Morales-Velázquez JL, Villaseñor-Ovies $P$, Muro-Cruz $D$. Classical fever of unknown origin (FUO): current causes in Mexico. Rev Invest Clin. 2005;57(6):762-9.

18. Crişan A, Nicoară E, Laza R. Considerations in prolonged febrile illness. Pneumologia. 2009;58(1):24-6, 28.

19. Ammari F. Fever of unknown origin in North Jordan. Trop Doct. 2006; 36(4):2513.

20. Mansueto $P$, Di Lorenzo G, Rizzo M, Di Rosa S, Vitale G, Rini G, Mansueto S, Affronti M. Fever of unknown origin in a Mediterranean survey from a division of internal medicine: report of 91 cases during a 12-year-period (1991-2002). Intern Emerg Med. 2008;3(3):219-25.

21. Zheng $M$, Lin $H$, Luo $S$, $X u L$, Zeng $Y$, Chen $Y$. Fever of unknown origin in the elderly: nine years experience in China. Trop Doct. 2008;38(4):221-2. 
22. Sipahi OR, Senol S, Arsu G, Pullukcu H, Tasbakan M, Yamazhan T, Arda B, Ulusoy S. Pooled analysis of 857 published adult fever of unknown origin cases in Turkey between 1990-2006. Med Sci Monit. 2007; 13(7): CR318-22.

23. Ma XJ, Wang AX, Deng GH, Sheng RY. A clinical review of 449 cases with fever of unknown origin. Zhonghua Nei Ke Za Zhi. 2004; 43(9):682-5.

24. Wei W, Hesheng L. Malignancy in Chinese adults presenting as fever of unknown origin. Int J Clin Pract. 2003;57(6):508-12.

25. Nakamuran J, Papac R, Ward R. Fever as initial manifestation of prostatic carcinoma. Urology. 1982;19:72-73

26. Gupta R, Setia N, Arora P, Singh S, Singh T. Hematological profile in pyrexia of unknown origin: role of bone marrow trephine biopsy. Hematology. 2008; 13(5): 307-12.

27. López Rodríguez M, Vázquez Muñoz E, Gómez Cerezo J, Suárez García I, Ríos Blanco JJ, Atienza Saura M, Barbado Hernández FJ. Cost-effectiveness of computerized axial tomography in the diagnosis of traditional clinical picture of fever of unknown origin. Rev Clin Esp. 2005; 205:19-23.
28. Görg C, Ozbatur $H$. The value of a standardized ultrasound in patients with fever. Ultraschall Med. 2009;30:396-400.

29. Torres KL, Flagueiredo DV, Zalis MG, Daniel-Riberio CT, Alecrim W, Ferreira-deCruz Mda F. Standardization of a very specific and sensitive single PCR for detection of Plasmodium vivax in low parasitized individuals and its usefulness for screening blood donors. Parasitol Res 2006; 98(7):519-24.

30. Singh UB, Bhanu NV, Suresh VN, Arora J, Rana $T$, Seth P. Utility of polymerase chain reaction in diagnosis of tuberculosis from samples of bone marrow aspirate. Am J Trop Med Hyg 2006; 75(5):960-3.

31. Nandagopal B, Sankar S, Lingesan K, Appu KC, Sridharan G, Gopinath AK. Prevalence of Salmonella typhi among patients with febrile illness in rural and peri-rural populations of Vellore district, as determined by nested PCR targeting the flagellin gene. Mol Diagn Ther 2010; 4(2): 107-12. 